\title{
Recent Advances in Optical Spectroscopic and Imaging Methods for Medicine and Biology
}

\author{
Yu Shang, ${ }^{1}$ Zhiyu Qian, ${ }^{2}$ Rickson C. Mesquita, ${ }^{3}$ and Mathieu Dehaes ${ }^{4}$ \\ ${ }^{1}$ University of Kentucky, Lexington, KY 40506, USA \\ ${ }^{2}$ Nanjing University of Aeronautics \& Astronautics, Nanjing 210016, China \\ ${ }^{3}$ University of Campinas, 13083-589 Campinas, SP, Brazil \\ ${ }^{4}$ University of Montréal, Montréal, QC, Canada H3T 1C5
}

Correspondence should be addressed to Yu Shang; yu.shang@uky.edu

Received 29 June 2016; Accepted 29 June 2016

Copyright (C) 2016 Yu Shang et al. This is an open access article distributed under the Creative Commons Attribution License, which permits unrestricted use, distribution, and reproduction in any medium, provided the original work is properly cited.

The optical spectroscopy and imaging have been emerging as fast and efficient methods to detect the material properties, especially in biological tissues. Basic and advance optical properties can be captured through investigation of light penetration, dispersion, and interaction with medium, permitting differentiation of the healthy and diseased tissues with a wide range of targets. Beyond the application on biological tissues, the spectroscopy/imaging methods are widely utilized in industrial field, for probing morphological information and internal defects of various components.

This special issue contains articles exploring the advances in spectroscopic/image methods for applications in biological tissues and industrial components. The utilized methodologies and key discoveries are summarized and highlighted in the following paragraphs.

Nasopharyngeal carcinoma (NPC) is a prominent malignant without obvious early symptom. The conventional histopathological examination of biopsy involves destruction and invasion of tissue from suspected lesions. To improve the survival rates of patient and promote the life quality, $\mathrm{Z}$. Xu et al. proposed a method of reflectance spectroscopy for early diagnosis of NPC in their article, entitled "Discrimination of Nasopharyngeal Carcinoma from Noncancerous Ex Vivo Tissue Using Reflectance Spectroscopy." The spectral system includes a xenon lamp, a spectrometer, and a computer. A band-pass filter (400-700 nm) was installed at the reflectance spectrograph entrance. The reflectance spectra data of nasopharyngeal tissue were collected from 18 noncancerous and 19 cancerous subjects, and multivariate statistical analysis was utilized to distinguish NPC from benign tissues. The experimental results exhibited a diagnostic sensitivity of $73.7 \%$ and a specificity of $72.2 \%$, verifying the clinical value of reflectance spectroscopy and advanced algorithm for NPC detection.

Detection of the pollutants caused by toxic metals and their effects on biological organisms is of great importance for disease prediction and human health. In the article entitled "Influence of Lead on the Interpretation of Bone Samples with Laser-Induced Breakdown Spectroscopy," A. Shahedi et al. developed laser-induced breakdown spectroscopy (LIBS) for tracing and identifying the elements available in bone sample. The LIBS system consists of a pulse laser at $1064 \mathrm{~nm}$ wavelength, a focusing lens, and CCD-array-based spectrograph. The experiments on rat thigh bone samples reveal that significant decrease in mineral $\mathrm{Mg}$ and $\mathrm{Ca}$ was observed due to lead consumption by the tissue, hence offering a prompt, nondestructive, and low-cost approach to probe the toxic substance associated with lead pollution.

Quick and accurate capture of the target with high speed is of importance for many dynamic applications. For this purpose, W. Lang et al. developed spectral identification system in their article entitled "High-Speed Target Identification System Based on the Plume's Spectral Distribution." The identification system consists of photometry with interference module, from which the interference data of motion target is acquired. By use of identification system, the spectral distribution data of the target can be obtained through smooth filtering, inversion, fast Fourier transform, and spectral calibration of the interference fringes. The characteristics that identify the target are finally determined 
by using recognition algorithm. Experiments demonstrate that the moving targets at less than $2000 \mathrm{~m} / \mathrm{s}$ can be accurately identified with the proposed spectral identification system.

Conventional approach to detect the internal defect of a component needs sensors to mark the "zero" positions, which is time-consuming. In the article entitled "Spatial Spectroscopy Approach for Detection of Internal Defect of Component without Zero-Position Sensors," Q. Wu et al. proposed a novelty approach of spatial spectroscopy for fast detection of internal defect of objects without zeroposition sensors. Specifically, the spatial variation wave of distance between the detecting source and object surface is analyzed, from which a periodical cycle is determined with the correlative approaches. Additionally, a wavelet method is adopted to reduce the noise of the periodic distance signal. Experiments on the component with round cross section and elliptical shape in axis demonstrate that spatial spectroscopy approach greatly saves the time and improves the detecting efficiency of internal defect in the components. This method can also be extended for use in biological tissues.

As a critical branch of diffuse optical tomography (DOT), the time-domain DOT exhibited a strong ability in quantifying various functional parameters such as hemoglobin and oxygen saturation. In the article entitled "Prospects on Time-Domain Diffuse Optical Tomography Based on Time-Correlated Single Photon Counting for Small Animal Imaging," Y. Bérubé-Lauzière et al. extensively reviewed the recent progress made to the high temporal resolution timedomain DOT system as well as its application on small animal molecular imaging. The review article focuses on introduction of the approaches to increase the imaging speed and increase image quality, both of which are highly crucial and challenging for time-domain DOT system. The hardware mostly affecting the spatial resolution and temporal resolution, such as single photon avalanche diode (SPAD) detectors and time-correlated single photon counting (TCSPC), is comprehensively discussed. Through exhibition of several experimental results, this review article further emphasizes the prospective of the next generation for time-domain DOT system.

We hope that this special issue will provide timely and sufficient information for the scientists and engineers working in field of optical spectroscopy and imaging, particularly for those with biomedical applications. We believe that the emerging spectroscopic/imaging approaches, spanning over both macro- and microlevel of tissue, will be greatly beneficial for diagnosis and therapy of various diseases.

\section{Acknowledgments}

We would like to thank all the authors for contributing their works to this special issue. We also appreciate the supports of the reviewers for their constructive critiques.

Yu Shang

Zhiyu Qian

Rickson C. Mesquita

Mathieu Dehaes 

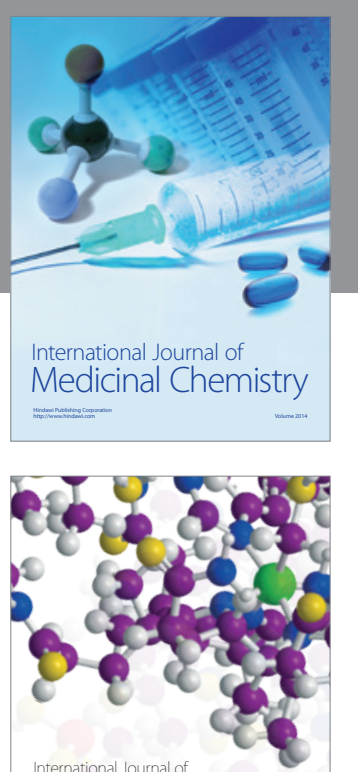

Carbohydrate Chemistry

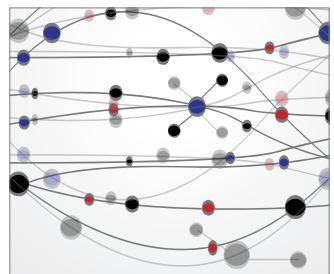

The Scientific World Journal
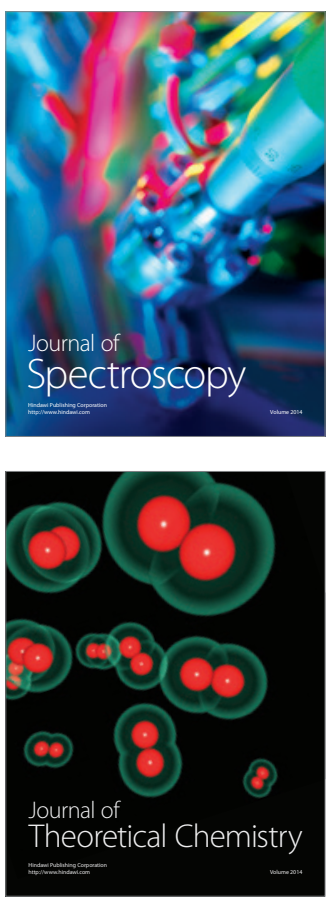
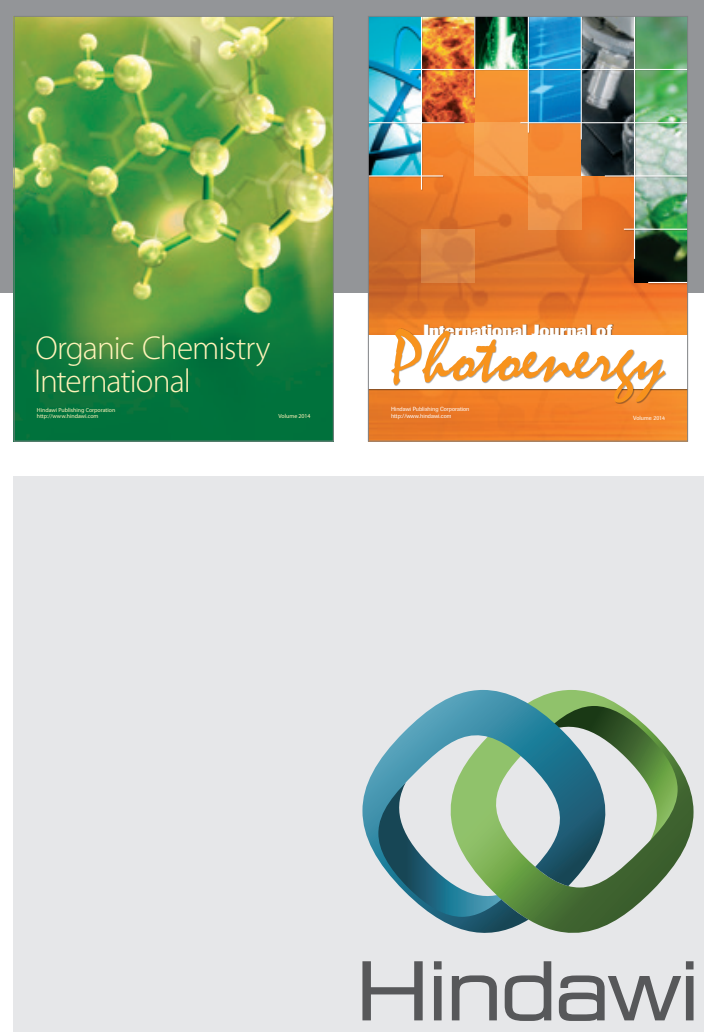

Submit your manuscripts at

http://www.hindawi.com

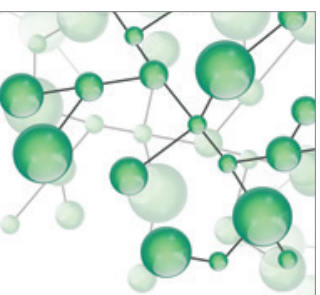

International Journal of

Inorganic Chemistry

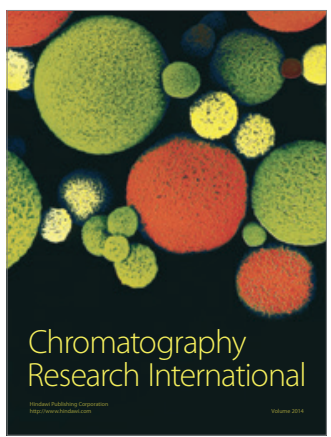

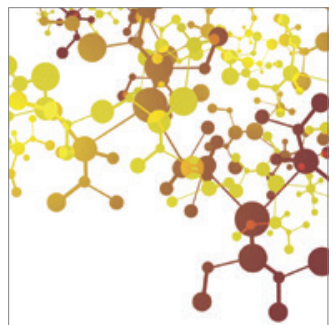

Applied Chemistry
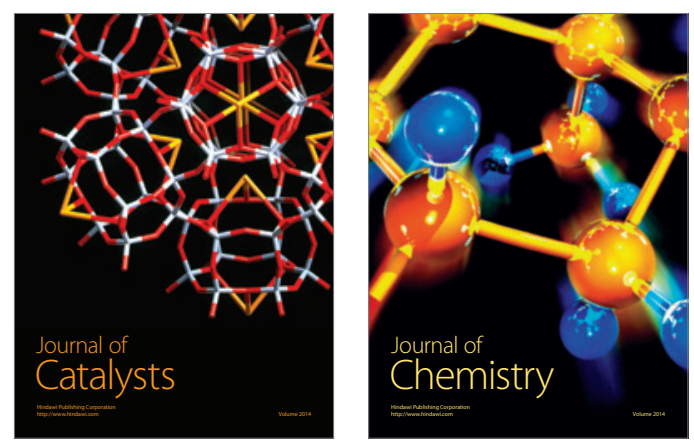
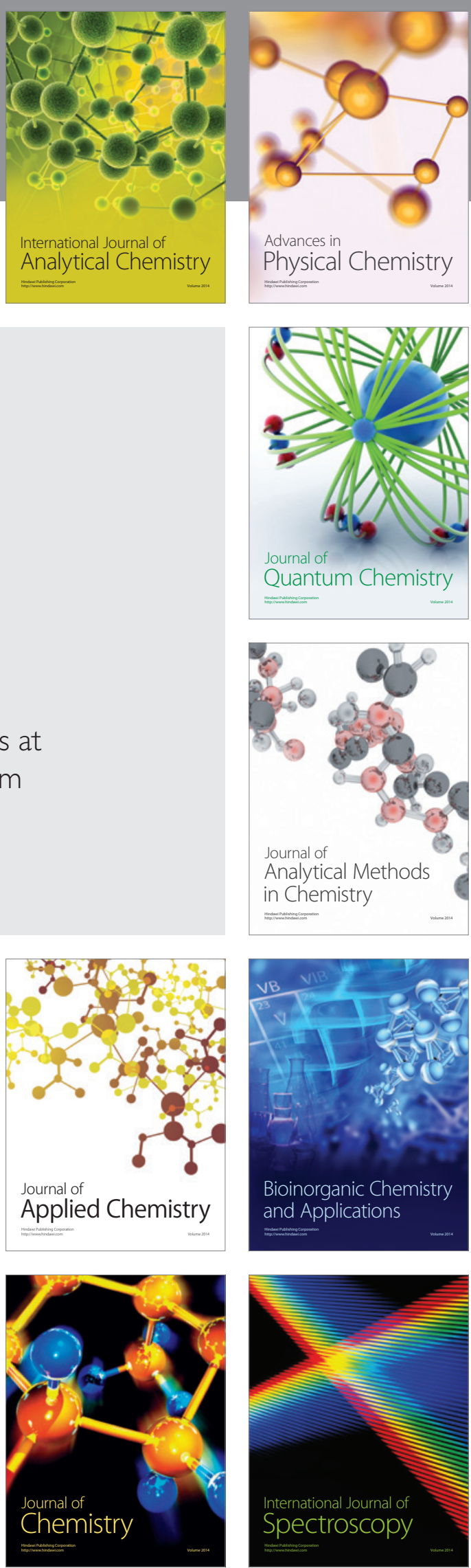